\section{METHANE EMISSIONS}

\section{Controls on Arctic methane}

Glob. Change Biol. http://doi.org/jw8 (2012)

Methane emissions from the Arctic have the potential to provide a strong positive feedback to the climate system, enhancing the degree of climate warming. However, the main physical and environmental controls on Arctic methane emissions remain unclear. This knowledge gap fundamentally limits the accuracy of predictions of methane emissions and ultimately the extent of climate change that can be expected in the Arctic.

In an effort to distil the state-of-theart in Arctic methane emissions research, David Olefeldt, from the Department of Integrative Biology, University of Guelph, Canada, and co-workers performed a synthesis of 65 studies involving over 300 Arctic sites where data on environmental and physical variables - including permafrost conditions - were available.

Their findings indicate that future changes in terrestrial high-latitude methane emissions will be more closely related to changes in moisture, soil temperature and vegetation composition than to the direct effects of permafrost thaw on organic matter availability.

\section{HUMAN IMPACTS}

\section{Climate-conflict nexus}

Proc. Natl Acad. Sci. 109, 18344-18349 (2012)

Previous studies have shown a positive impact of climatic variability on the risk of conflict in sub-Saharan Africa, with temperature as the strongest driver of violence. However, the lack of thorough geographical considerations challenges those results.

John O'Loughlin of the University of Colorado, Boulder, USA, and colleagues examined climate-conflict relationships with, for the first time, a geographically disaggregated approach. Using a conflict database that contains 16,359 individual geo-located violent events for East Africa from 1990-2009, they considered a wide range of socio-economic, geographic and political factors at a $1^{\circ}$ gridded resolution. Unlike earlier studies, they found that after controlling for the non-geographic factors - precipitation levels that are two standard deviations above the long-term average decrease the risk of violence by $30.3 \%$, whereas drier and normal periods have no effect. Results also show that temperatures two standard deviations higher than the average raise the risk of violence to $29.6 \%$, with normal and cooler temperatures not affecting incidence of violence.

$M C$

\section{OCEAN ACIDIFICATION}

Aragonite shell damage

Nature Geosci. 5, 881-885 (2012)

Ocean models predict that the confluence of natural (deepwater ventilation) and anthropogenic (ocean acidification) factors may cause the upper layers of the Southern Ocean to become undersaturated in aragonite - a form of calcium carbonate by 2050 . Such undersaturation is likely to affect aragonite-shelled organisms, which can dominate surface water communities in polar regions and constitute an important component of the oceanic carbon system.

Nina Bednaršek, from the British Antarctic Survey, Cambridge, UK, and co-workers sampled from surface waters with low aragonite-saturation levels $\left(\Omega_{\mathrm{A}} \sim 1\right)$ in the Southern Ocean, and compared the shell structure of a pteropod (Limacina helicina antarctica) - a species of planktonic mollusc - with specimens from aragonite-supersaturated regions

Ozone and particulate matter can accumulate in still atmospheric conditions and lead to harmful air quality. Climate change is altering global atmospheric circulation and precipitation patterns, which regulate the frequency of air stagnation.

Daniel Horton, of Stanford University USA, and co-workers used a multimodel ensemble of global climate models to investigate the response of the National Climatic Data Center air-stagnation index to global warming, up to the end of this century. Their results show the highly industrialized and highly populated regions of the eastern United States, Mediterranean Europe and eastern China have atmospheric conditions that are particularly vulnerable to global warming and increased stagnation of air.

Air quality is an area of concern as health can be greatly affected. This study shows that air quality could be affected by changing atmospheric conditions as a result of global warming. Further work is necessary to quantify the impacts of global warming on air quality, and the consequences for health. using scanning electron microscopy. They found severe levels of shell dissolution in the undersaturated samples.

These findings in live pteropods in their natural environment validate laboratory based studies that showed the potential vulnerability of L. helicina antarctica to ocean acidification. Furthermore, this study demonstrates that ocean acidification is already having an impact - ahead of many aragonite-saturation projections - indicating that regional declines in pteropod populations may occur sooner than previously predicted.

\section{PALEOCLIMATE}

\section{Reconstructing temperature}

Geophys. Res. Lett. http://doi.org/jw9 (2012)

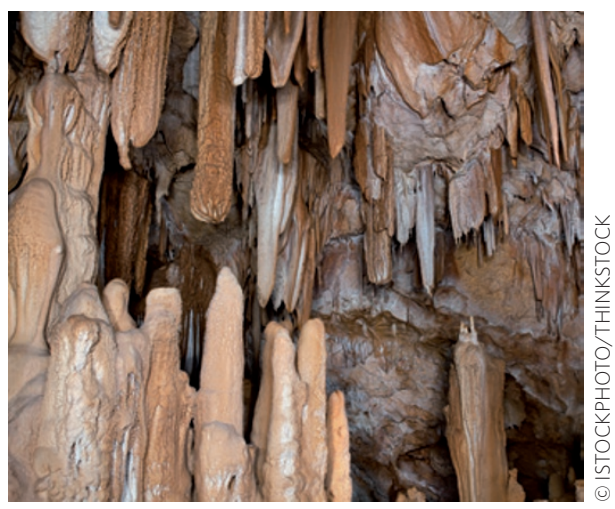

The global surface temperature timeseries which is thermometer based - provides key evidence for global warming. However, uncertainties associated with this record are large, and it also suffers from limitations in temporal and spatial coverage.

A new Paleo Index has been compiled by David Morrill Anderson, of the National Ocean and Atmospheric Administration's National Climate Data Center, USA, and colleagues. The index was derived from 173 temperature-sensitive proxy timeseries including: corals, ice cores, speleothems (mineral deposits in caves), lake and ocean sediments and historical documents. It extends back to 1730 , with a global distribution. The Index shows a significant upward trend for 1880-1995, as well as accelerated warming after 1980 .

Comparing the Paleo Index with global surface temperature shows similar small-scale trends, providing independent evidence of the warming observed. These findings support the thermometer data, increasing confidence in the accuracy of the global surface temperature timeseries.

Written by Alastair Brown, Monica Contestabile and Bronwyn Wake. 